\title{
CMOS APS in pre-clinical science: next generation disruptive technology for multi-modality imaging
}

\author{
M. Esposito ${ }^{1}$, A. Bailey ${ }^{2}$, J. Newcombe ${ }^{3}$, T. Anaxagoras ${ }^{4}$, N. M. Allinson ${ }^{5}$ and K. Wells ${ }^{1}$
}

\begin{abstract}
A new large area CMOS Active Pixel Sensor has been developed as single platform technology to be used across a range of ionizing and non-ionizing imaging applications in preclinical science, ranging from imaging of protein sequences to functional analysis of radio-labeled tissue sections. We present the first images of chemiluminescence detection in western blotting with a room temperature CMOS APS. Detection performance in western blotting have been compared with the gold standard detection medium, film emulsion, showing higher dynamic range and sensitivity with this new device. We also report on our first images of $\left[{ }^{125} \mathrm{I}\right]$ Epibatidine autoradiography of brain sections using a novel large area CMOS APS.
\end{abstract}

Index Terms-Pre-clinical imaging, Life science imaging applications, Autoradiography, Chemiluminescence detection, CMOS, APS, Large area image sensors, semiconductor devices.

\section{INTRODUCTION}

$\mathbf{P}$ RE-CLINICAL science utilizes structural, functional, metabolic and molecular probes for a wide range of applications including characterizing disease phenotypes and molecular mechanisms, evaluating drug efficacy and identifying potential bio-markers, in order to drive advances in healthcare. Such activity relies on a wide range of techniques requiring different probe detection methods, such as $\beta^{-}$or $\gamma$ autoradiography of specifically labeled tissue, ex-vivo positron autoradiography of small animals sections for comparative analysis with PET imaging, optical detection of immunodetected proteins, DNA and RNA labeled with chemiluminescent or fluorescence probes and UV shadow imaging for DNA analysis.

The most commonly used detection medium in this field is film emulsion, although other detection and imaging media are also used such as phosphor plate technology, amorphous imaging plates, and specialist systems using cooled camera technology. Film offers an unbeatable spatial resolution due to its fine granularity, but presents severe drawbacks (limited dynamic range, non-linear response and low sensitivity) which strongly limits the imaging performance and entails lengthy exposures: a significant bottleneck in the routine experimental

\footnotetext{
${ }^{1}$ Centre for Vision, Speech and Signal Processing, Faculty of Engineering and Physical Sciences, University of Surrey, Guildford GU2 7XH,U.K. (email:m.esposito@surrey.ac.uk ).

${ }^{2}$ Department of Biochemistry and Physiology, Faculty of Health and Medical Sciences, Institute of Biosciences and Medicine, University of Surrey, Guildford, GU27XH, U.K.

${ }^{3}$ Department of Microbial and Cellular Sciences, Faculty of Health and Medical Sciences, University of Surrey, Guildford, GU27XH, U.K.

${ }^{4}$ ISDI Ltd (Image sensor design and innovation), Oxford, OX4 1YZ, U.K

${ }^{5}$ School of Computer Science, University of Lincoln, Lincoln, LN6 7TS, U.K.
}

work-flow. Moreover, in those applications, where it is not possible to asses the a priori uptake of ligands in the biological sample of interest, an expensive and time consuming procedure of multiple exposures is required.

A range of alternative technologies have been proposed to address these limitations, including Multi-Wire Proportional Chambers (MWPC) [1], micro-strip detectors [2], [3], gaseous detector [4], phosphor plates [7], Micro-Channel Plates (MCPs) [5], [6] and solid-state commercial and hybrid direct-detection detectors [8], [9], [10]. Of these, only phosphor plates and systems based on cooled CCD cameras have produced significant impact, albeit for a limited range of applications largely due to the particular limitations of the technology. Direct detection methods with pixellated CCD/CMOS/hybrid sensors have been frequently handicapped by modest imaging areas $\left(\sim\right.$ few $\left.\mathrm{cm}^{2}\right)$. This places an upper bound on throughout potential, off-setting the benefits of any performance gain, and so limiting application beyond initial 'point source' proof-of-concept.

Moreover a number of digital imaging systems for nonionizing imaging applications in life science have being developed in the last two decades, mainly based on the use of CCDs [11] [12] [13] [14] and single avalanche diodes [15]. Even so these systems exhibit serious shortcomings in imaging performance, such as a low frame rate due to the inherent sequential read-out, require operation at low temperature to achieve a reasonable noise level or suffer from poor geometrical efficiency due to the optics involved.

In this paper we address these issues by presenting first results demonstrating the efficacy of using a wafer-scale CMOS imaging sensor as a viable single platform technology that can be used across a range of pre-clinical ionizing and non-ionizing imaging applications. We present first results of imaging an electrophoresis sample, obtained using Western Blotting methods, and labelled with Luminol, an optical probe. We also present our first beta-labeled autoradiography results using a large area CMOS APS, whee LaTeX engine places floats (figure, tables, etc.). When typesetting large documents that contain a lotrein several full size microscope slides can be placed directly on the sensor surface without further preparation.

\section{Multi-Modality IMAGing SYSTEM}

The multi-modality imaging system presented here is based on the use of a large area CMOS Active Pixel Sensor, called Dynamic Range Adjustable for Medical Imaging Technology (DynAMITe), and constructed in a $0.18 \mu \mathrm{m}$ CMOS process 


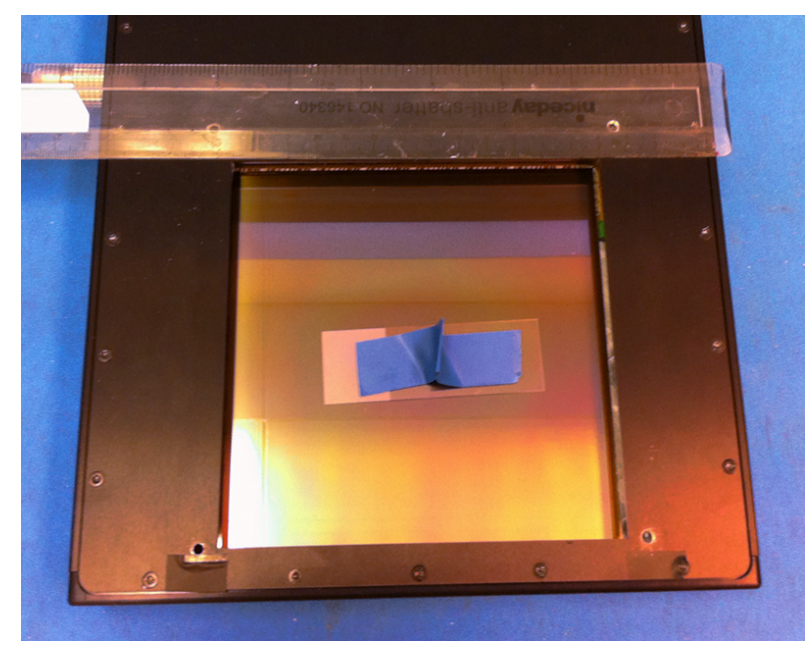

Fig. 1. The multi-modality imaging system showing an imaging area of 12.8 $\mathrm{cm} \times 13.1 \mathrm{~cm}$. A microscope slide $(75 \mathrm{~mm} \times 25 \mathrm{~mm})$ is placed on the sensor active area.

by a reticule stitching technique for a total active area of 12.8 $\mathrm{cm} \times 13.1 \mathrm{~cm}[16]$.This sensor is also two sided edge buttable, facilitating expansion up to approximately $26 \times 26 \mathrm{~cm}^{2}$.

The imaging system is illustrated in fig. 1 . The APS detector used here relies on the concept of using different size diodes meshed in the same pixel array, thus realizing two imagers in one. The detector consists of fine-pitch grid diodes, offering intrinsic low noise and high spatial resolution, and largepitch grid diodes, offering a high dynamic range. The inbuilt dual pixel resolution of the Dynamite sensor can thus be optimally reconfigured for different imaging applications [16]. The imaging system is placed in a light tight enclosure and operated at room temperature. Samples, using both ionizing and non-ionizing probes, are placed in direct contact with the detector surface and imaged at $1 \mathrm{fps}$.

As samples used in pre-clinical science usually produce a low intensity signal compared with the detector dark signal, an $a d$ hoc procedure for per-pixel on-line dark correction has been developed, based on both temporal shift of the reference dark signal and per-pixel variation. Additionally a windowing technique, consisting of adaptive high and low threshold levels, has been used to increase the rejection rate of false events.

\section{Western blotting of E. Coli Proteins}

\section{A. Western blotting}

Escherichia coli total proteins were denaturated by heating at high temperature and subsequently loaded onto a SDSPAGE 12\% Acrylamide gel in amount of 5, 10 and $15 \mu$ l. Protein gel was subjected to electrophoresis for 1 hour by an electric potential difference of $200 \mathrm{~V}$. The electric field resulting from the potential difference allowed proteins to migrate through the gel, which behaves like a sieving medium, with a velocity depending upon their charge and molecular weight. Separated proteins were then transfered to a Polyvinylidine Fluoride (PVDF) membrane (Hybond - LEP membrane, Amersham Biosciences) by overnight electroblotting in order to be immuno-detected.

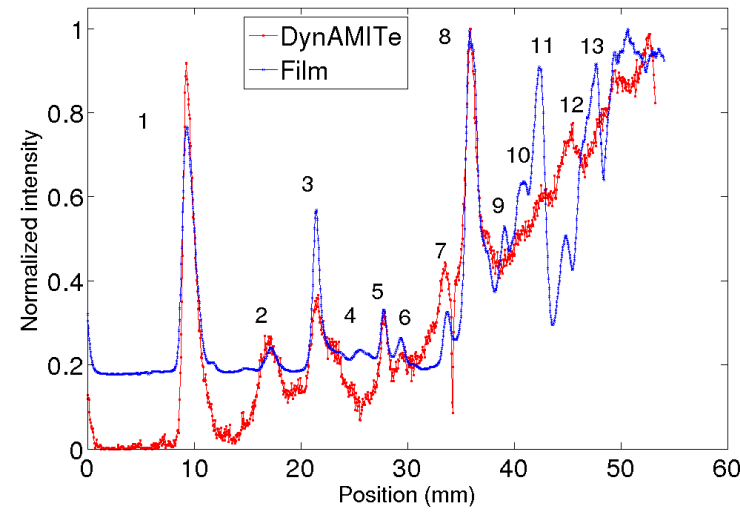

Fig. 3. Intensity profile across the lines containing $5 \mu \mathrm{l}$ of $E$. coli proteins (see fig. 2) displayed for the DynAMITe detector (red line) using $1 \mathrm{~s}$ exposure time and for film emulsion (blue line) with $1 \mathrm{~min}$ exposure time. Detected peaks are numbered.

Immuno-detection is a multi-step process: in order to be detected proteins bind a specific primary antibody, which, in turn, binds a secondary specific antibody. Secondary antibodies are bound to $\mathrm{H}_{2} \mathrm{O}_{2}$ in order to emit chemiluminescence light when in contact with Luminol, according to the following chemical reaction:

Luminol $+\mathrm{H}_{2} \mathrm{O}_{2} \rightarrow 3-\mathrm{AP} A[\diamond] \rightarrow 3-\mathrm{APA}+\gamma(425 \mathrm{~nm})$.

Samples were incubated with Bovine Serum Albumin, as a blocking reagent to prevent from non-specific binding and then washed with human serum containing primary antibodies and then with secondary antibodies, anti-human IgG HRP antibody (A8667, Sigma-Aldrich). In order to allow chemiluminescence emission, the membrane was washed with Lumi-Light Western Blotting Substrate (Roche Applied Science).

\section{B. Imaging of immuno-detected proteins}

In Fig. 2 we present the first images of western blotting of proteins via immuno-detection with a room temperature CMOS APS. Membrane sections containing 5, 10 and $15 \mu \mathrm{l}$ of proteins were imaged with both the DynAMITe detector and film emulsion, for a cumulative integration time of $30 \mathrm{~s}$, 1 and $3 \mathrm{~min}$. Film images are strongly affected by saturation in the longer exposure images, whereas the DynAMITe detector remains unsaturated due to a larger dynamic range. An intensity profile across the lines containing $5 \mu \mathrm{l}$ of proteins is plotted in fig. 3 for both detection systems, obtained with $1 \mathrm{~s}$ exposure time for DynAMITe and $1 \mathrm{~min}$ exposure time for film emulsion. Detected peaks, numbered in figure, are comparable in number for both systems with an exposure time ratio of 60 .

\section{IMAGING OF $\left[{ }^{125}\right.$ I]EPIBATIDINE LABELED BRAIN SECTIONS}

\section{A. $\left[{ }^{125}\right.$ I]Epibatidine labelling of brain tissue sections}

Mice brain slices were labeled with $\left[{ }^{125}\right]$ Epibatidine to undertake addiction studies on the nicotine brain receptors a4 $\beta 2$-nAChR. In fact $\left[{ }^{125}\right.$ I]Epibatidine binds with high affinity 


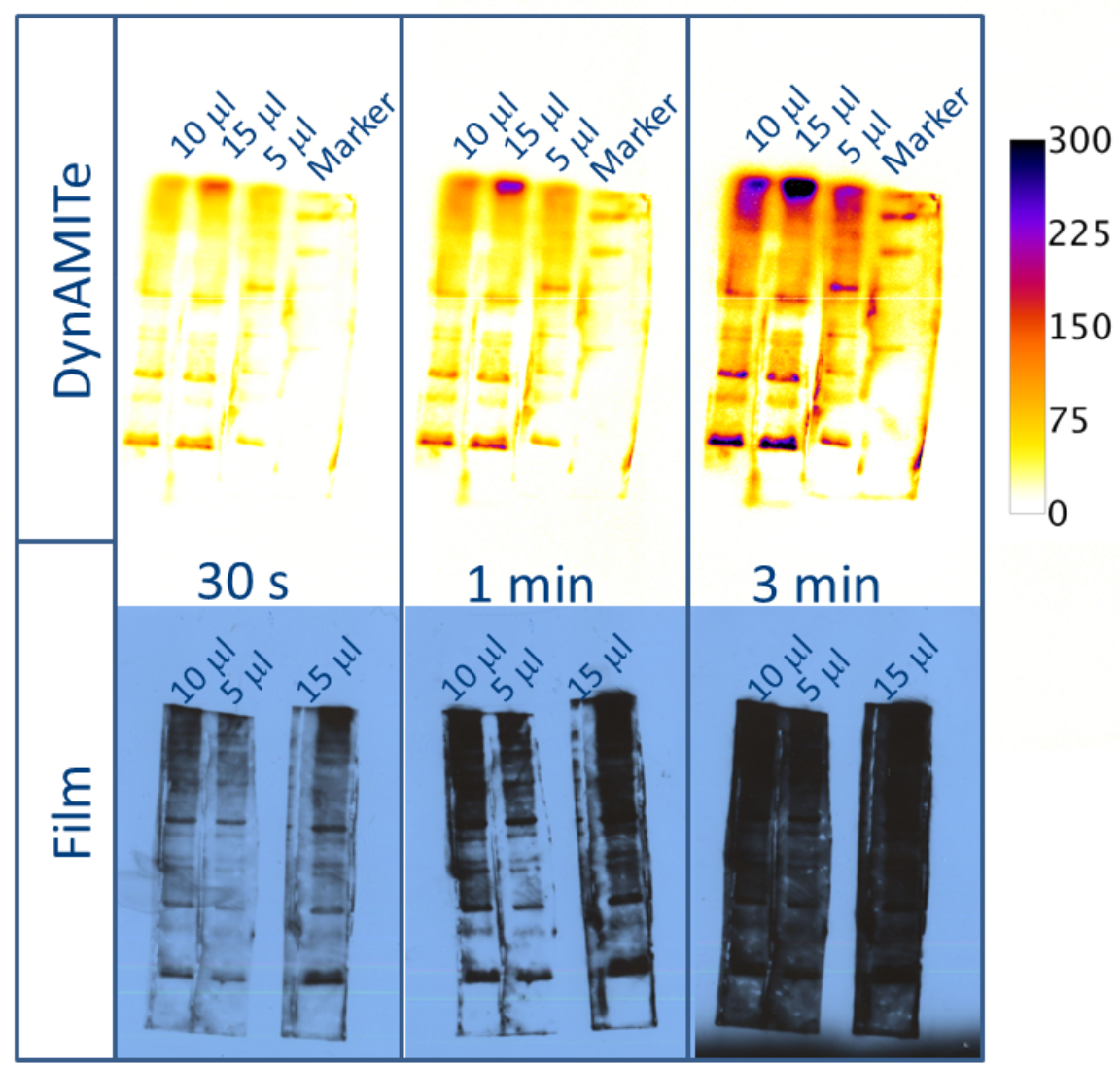

Fig. 2. First images of optical labeled protein sequence imaged using the multi-modality imaging system. Samples were imaged with both the DynAMITe detector and film emulsion for $30 \mathrm{~s}, 1 \mathrm{~min}$ and $5 \mathrm{~min}$ exposure time.

several nicotine receptors. Brain sections were incubated for $2 \mathrm{~h}$ with $\left[{ }^{125} \mathrm{I}\right]$ Epibatidine, with a solution of $\left[{ }^{125} \mathrm{I}\right]$ Epibatidine and Cytosine to evaluate competitive ligands and with nicotine salts for evaluation of non specific binding.

Samples were imaged after 16 days from the sample preparation with a residual activity of $83 \%$ following film exposure, given the ${ }^{125} \mathrm{I}$ half life $T=59.4 d$.

\section{B. ${ }^{125}$ I autoradiography}

Our first images of ${ }^{125}$ I label tissue section acquired with a room temperature CMOS APS are displayed in fig. 4. Images of five slides labeled A-E, containing 12 tissue slices each, are displayed for $1 \mathrm{~h}, 5 \mathrm{~h}$ and $9 \mathrm{~h}$ exposure in insets $a, b, c$ of fig 4 respectively, together with a color scale calibrated in Counts pixel $^{-1}$.

Fig. 5 shows the linearity plot for the imaged samples. For each slide (A-E) signal detected in one of the tissue slices has been plotted as function of the exposure time. The background level for this experiment is represented with a dotted line in figure. For samples C, D and E, which present a higher activity, signal shows a linear behavior $\left(\mathrm{R}^{2}>0.99\right)$ in all the time range investigated. Samples A and B, characterized by a lower activity, have a signal level comparable with the background level after 1 hour exposure. The linear fit for lower activity samples (slides A and B) has been then performed in the range 2-9 $\mathrm{h}$ exposure time, resulting in a coefficient of determination
$\mathrm{R}^{2}>0.99$. Hence all the samples investigated in this work present a linear behavior with time, above the background level.

\section{CONCLUSIONS AND FUtURE WORK}

We have proposed large area CMOS APS as a multimodality imaging platform for use in pre-clinical science. The dual camera features of the Dynamite sensor which delivers two inherently different spatial resolutions, noise and saturation performance in the same pixel array, is user-configurable to particular imaging applications. We have demonstrated for the first time direct 'contact print' imaging of radioactive and optical labeled biological samples on a large imaging area, demonstrating its potential application to a broad range of ionizing and non-ionizing imaging probes.

\section{ACKNOWLEDGMENT}

This work was supported by the RC-UK Multidimensional Integrated Intelligent Imaging Plus (MI-3 Plus) programme (EPSRC Ref. EP/G037671/1).

\section{REFERENCES}

[1] Y. Petagnief et al., "Quantitative autoradiography using a radioimager based on a multiwire proportional chamber", Phys. Med. Biol., 43, 36292638, 1998

[2] B. Sanghera et al. Digital beta autoradiography using silicon microstrip detectors, Nucl. Instr. Meth. A(310) (1997) 455-9. 

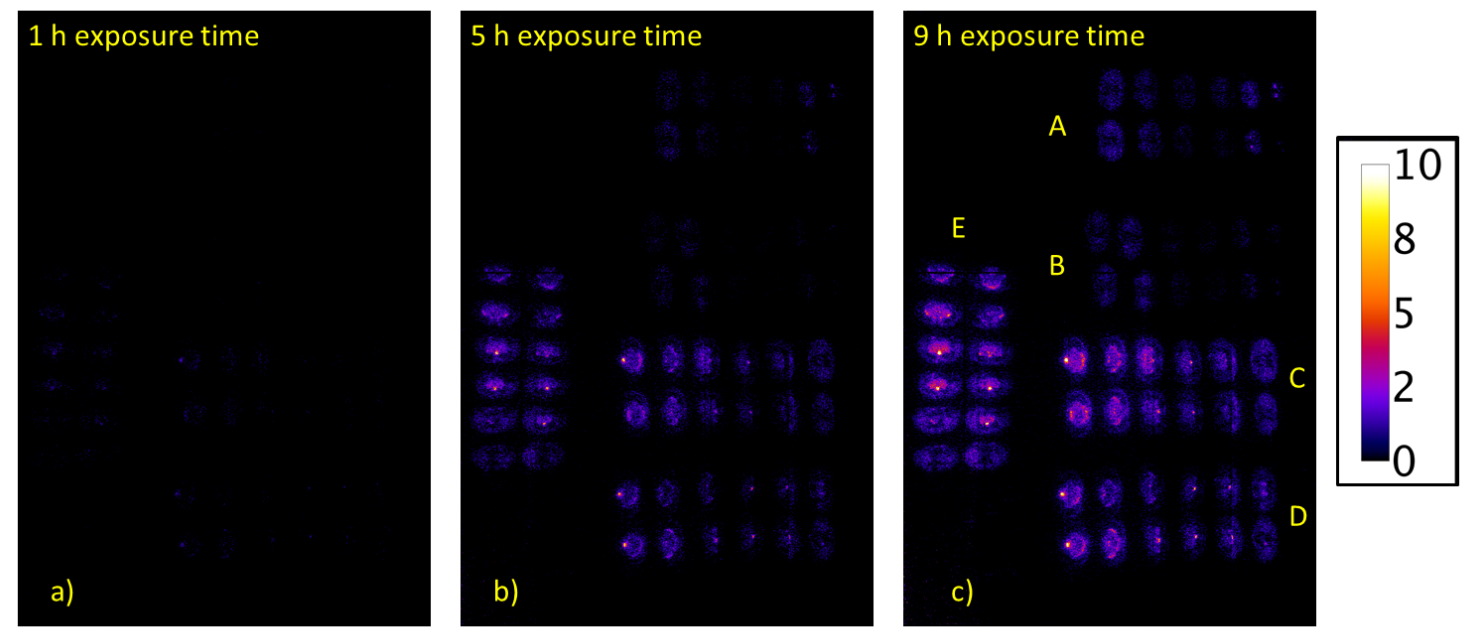

Fig. 4. $\left[{ }^{125} \mathrm{I}\right]$ Epibatidine brain tissue sections imaged with the DynAMITe detector with $1 \mathrm{~h} a, 5 \mathrm{~h} b$ and $9 \mathrm{~h} c$ exposure time. A color scale calibrated in Count pixel $^{-1}$ is also displayed.

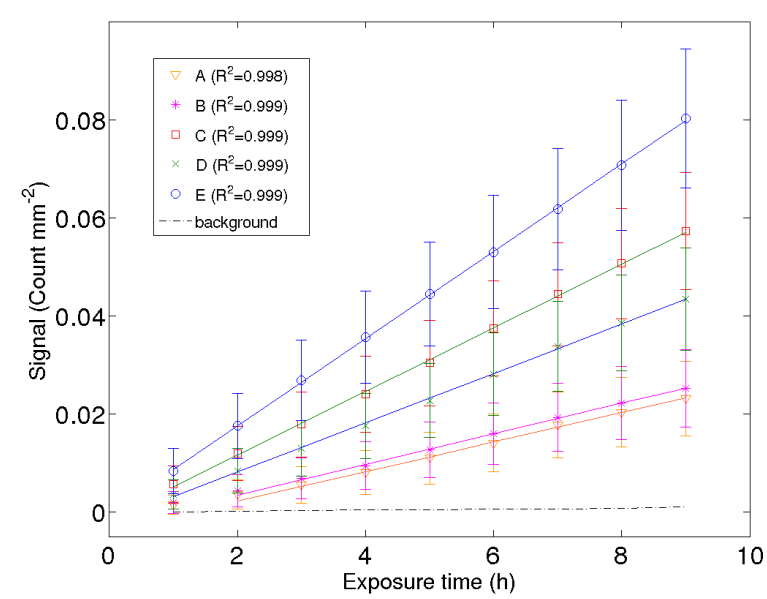

Fig. 5. Signal plotted versus exposure time for each sample of fig. 4 (AE). A linear fit for each of the samples is also displayed together with the determination coefficient $\mathrm{R}^{2}$. The background level for this experiment is represent with a dotted line.

[3] M. Overdick et al. A bioscope system using double-sided silicon strip detectors and self-triggering read-out chips, Nucl. Instr. Meth. A(392) (1997) 173-7.

[4] J. Donnard et al. The micro-pattern gas detector PIM: a multi-modality solution for novel investigations in functional imaging, Nucl. Instr. Meth. A(610) (2009) 158-60.

[5] J. P. Lees et al. Direct beta autoradiography using MCP detectors, Nucl. Instr. Meth. A(392) (1997) 349-53.

[6] J. P. Lees et al. Microchannel plate detectors for 14C autoradiography, IEEE Trans. Nucl. Sc. 45 (1998) 1288-92

[7] N. Barthe et al., "Recent technology developments on high resolution beta imaging for quantitative autoradiography and double labeling applications", Nucl. Instr. Meth., A 527, 41-5, 2004

[8] J. Cabello et al., "Digital autoradiography using room temperature CCD and CMOS imaging technology.', Phys. Med Biol., 52, Pag 4993-5011 (2007)

[9] G. Mettivier et al. First images of a digital autoradiography system based on a Medipix 2 hybrid silicon pixel detector, Phys. Med. Biol. 48 (2003) 173-81.

[10] M. Esposito et al., "14C autoradiography with an energy-sensitive silicon pixel detector", Phys. Med. Biol., 56, 1947-1965, 2011

[11] A. R. Mahon et al., "A CCD based system for the detection of DNA in electrophoresis gel by UV absorption", Phys. Med. Biol., 44, 1529-41, 1999

[12] FluorChemQ System, Quantitative Western Blot imaging, Cell Biosciences, online,http://www.cellbiosciences.com/fluorchemq.html

[13] Ettan DIGE, GE Healthcare, online,http://gelifesciences.com

[14] Chemidoc XRS + System, online, http://www.bio-rad.com

[15] M. Gersbach et al., "A room temperature CMOS single photon sensor for cheiluminescence detection", presented at Internation Conference on Miniaturized Systems for Chemistry and Life Science (MicroTas), November 2006.

[16] M. Esposito et al., "DynAMITe: a wafer scale sensor for biomedical applications", Journ. Instr., 6 C12064, 2011. 